\title{
Restoration of outer segments of foveal photoreceptors after resolution of central serous chorioretinopathy.
}

\section{$\operatorname{AUTHOR}(\mathrm{S}):$}

Ojima, Yumiko; Tsujikawa, Akitaka; Yamashiro, Kenji; Ooto, Sotaro; Tamura, Hiroshi; Yoshimura, Nagahisa

\section{CITATION:}

Ojima, Yumiko ...[et al]. Restoration of outer segments of foveal photoreceptors after resolution of central serous chorioretinopathy.. Japanese journal of ophthalmology 2010, 54(1): 55-60

\section{ISSUE DATE:}

2010-01

URL:

http://hdl.handle.net/2433/108271

\section{RIGHT:}

(C) 2010 Japanese Ophthalmological Society (JOS).; This is not the published version. Please cite only the published version.; この論文は 出版社版でありません。引用の際には出版社版をご確認ご利用くださ い。 


\section{RESTORATION OF OUTER SEGMENTS OF FOVEAL PHOTORECEPTORS}

\section{AFTER RESOLUTION OF CENTRAL SEROUS CHORIORETINOPATHY}

YUMIKO OJIMA, MD, AKITAKA TSUJIKAWA, MD, KENJI YAMASHIRO, MD,

5 SOTARO OOTO, MD, HIROSHI TAMURA, MD, NAGAHISA YOSHIMURA, MD

From the Department of Ophthalmology and Visual Sciences, Kyoto University

Graduate School of Medicine, Kyoto, Japan.

10 The authors have no financial interest in the materials or devices mentioned in this article.

Correspondence to: Akitaka Tsujikawa, MD, Department of Ophthalmology, Kyoto University Graduate School of Medicine, Sakyo-ku, Kyoto 606-8507, Japan; fax:

15 +81-75-752-0933; e-mail: tujikawa@kuhp.kyoto-u.ac.jp 


\section{Abstract}

Purpose: To study morphologically and functionally the prognosis of damaged outer segments of the foveal photoreceptor layer in eyes with resolved central serous chorioretinopathy (CSC).

$5 \quad$ Methods: We studied retrospectively the medical records of 70 patients (74 eyes) with resolved CSC. Optical coherence tomography was used to detect the junctions between inner and outer segments of the photoreceptors (IS/OS) as a hallmark of integrity of the outer photoreceptor layer.

Results: In 53 eyes (71.6\%), the IS/OS line was detected clearly beneath the fovea

10 immediately after resolution of the retinal detachment, with good visual acuity (VA). In the remaining 21 eyes (28.4\%), however, the foveal IS/OS line could not be detected shortly after resolution of CSC, and VA was variable at that time, ranging from 0.1 to 1.5 (median, 0.9 ). In 4 of 15 eyes with follow-up examinations, the foveal IS/OS line was not detected throughout follow-up, and vision in these eyes remained

15 poor. However, nine eyes showed recovery of the foveal IS/OS line during follow-up, and these eyes showed substantial visual recovery.

Conclusions: Immediately after resolution of active CSC, even though the IS/OS line is not detected beneath the fovea, it often shows restoration over time, with visual recovery. 
Restoration of Photoreceptors in CSC. Ojima $Y$, et al. Page 3

Key words: central serous chorioretinopathy, optical coherence tomography, outer segment, photoreceptor layer. 


\section{Introduction}

Central serous chorioretinopathy (CSC) is characterized by focal serous retinal detachment in the macular area. ${ }^{1}$ In most eyes, the acute CSC resolves

5 spontaneously or immediately after laser photocoagulation or photodynamic therapy, and these eyes achieve full recovery of visual acuity (VA)., ${ }^{1,2}$ However, some eyes show a poor visual recovery in spite of complete resolution of the serous detachment. $^{3-6}$

Recently, optical coherence tomography (OCT) has contributed to our

10 understanding of the importance of the outer aspect of the foveal photoreceptor layer in visual function in macular diseases. ${ }^{7-10}$ After resolution of the ocular manifestations associated with CSC, previous examinations with use of Stratus OCT showed that foveal attenuation in the outer retina is associated with poor visual prognosis. ${ }^{4,5}$ More specifically, Eandi et al ${ }^{3}$ have shown an association between the

15 lack of an external limiting membrane, or a lack of the junctions between inner and outer segments of the photoreceptors (IS/OS) beneath the fovea, and decreased VA in resolved CSC. Recently, using spectral-domain OCT in eyes with resolved CSC, Ojima et $\mathrm{al}^{6}$ reported that a large defect of the outer segments in the foveal photoreceptor layer was correlated with poor VA. 
Visual prognosis of acute CSC is relatively good. In the clinical setting, however, some eyes with chronic or recurrent CSC have shown poor VA immediately after resolution of the serous detachment, often with an indistinct or defective IS/OS line beneath the fovea., 6 Longstanding detachment of the retina may cause apoptotic

5 cell death or a loss of the outer segments of the foveal photoreceptor cells. ${ }^{11}$ These eyes would not be expected to show recovery of the IS/OS line or subsequent visual recovery. ${ }^{6}$ To date, however, little information is available on the recovery of these damaged foveal photoreceptors in eyes with resolved CSC. ${ }^{12}$ Accordingly, the current study was designed to review OCT images obtained from eyes with resolved

10 CSC in order to study the prognosis of damaged outer segments of the foveal photoreceptor layer and its association with VA. 


\section{Subjects and Methods}

We retrospectively reviewed the medical records of 70 consecutive patients (74 eyes) with resolved CSC who were examined at the Macula Service, Department of

5 Ophthalmology, Kyoto University Hospital, between June 2005 and May 2008. At the initial visit, all patients showed active CSC with serous retinal detachment in the posterior pole. All patients had undergone a comprehensive ophthalmologic examination, including measurement of best-corrected VA, determination of intraocular pressure, indirect ophthalmoscopy, slitlamp biomicroscopy with a contact

10 lens, and OCT. For the current study, we excluded those eyes in which we could not confirm with OCT that serous retinal detachment associated with CSC affected the fovea at the initial visit, and those in which serous retinal detachment had not resolved completely during the follow-up period. Best-corrected VA was measured with a Landolt chart. After fundus photographs were taken, fluorescein angiography (FA) and indocyanine green angiography were performed on each patient using a confocal laser scanning system (HRA-2, Heidelberg Engineering, Dossenheim, Germany). This study was approved by the Institutional Ethics Committee and adhered to the tenets of the Declaration of Helsinki.

The diagnosis of CSC was based on results of the fundus examination, FA, and 
indocyanine green angiography. At the initial visit, all eyes showed focal serous retinal detachment by fundus examination and OCT. In most cases, FA showed leakage from the retinal pigment epithelium. Eyes with other macular abnormalities, such as neovascular maculopathy (i.e., idiopathic choroidal neovascularization,

5 polypoidal choroidal vasculopathy, age-related macular degeneration, or other secondary choroidal neovascularization), intraocular inflammation, posterior segment tumor, drusen, and any other condition that could cause a serous retinal detachment unrelated to CSC were excluded from the current study.

At each follow-up visit, all patients had undergone a comprehensive ophthalmologic examination, including measurement of best-corrected VA, determination of intraocular pressure, indirect ophthalmoscopy, slitlamp biomicroscopy with a contact lens, and OCT examination. We used time-domain OCT (Stratus OCT3000, Carl Zeiss, Dublin, CA; OCT ophthalmoscope C7, Nidek, Gamagori, Japan) and spectral-domain OCT (3D OCT-1000, Topcon, Tokyo, Japan; RTVue-100, Optovue,

15 Fremont, CA; Spectralis HRA+OCT, Heidelberg Engineering; Cirrus HD-OCT, Carl Zeiss). OCT images were reviewed in an unmasked fashion by two retinal specialists.

Statistical analysis was performed using software designed for this purpose (StatView, version 5.0; SAS Institute, Cary, North Carolina). To compare the 
difference in VA, best-corrected VA measured with a Landolt chart was converted to a logarithm of the minimal angle of resolution (logMAR). A $P$ value of less than 0.05 was considered to be statistically significant. 


\section{Results}

In the current study, 74 eyes of 70 patients (59 men and 11 women) with resolved CSC, ranging in age from 25 to 73 years ( $48.6 \pm 11.1$ years), were examined. At the

5 initial visit, all eyes showed focal serous retinal detachment in the macular area, involving the fovea. Of the 74 eyes with active CSC, 43 were in the acute phase, 10 were chronic, and 21 had a recurrence. Serous detachment was resolved with focal photocoagulation in 18 eyes, photodynamic therapy in 10 eyes, and without any treatment in 50 eyes. Mean follow-up period of these patients was $14.1 \pm 13.6$ months.

At the initial visit, all eyes showed a hyporeflective space corresponding to subretinal fluid beneath the fovea. Neurosensory retina overlying the serous retinal detachment showed neither macular oedema nor severe retinal thinning. The line of external limiting membrane was often seen clearly--not only in the attached retina but also in the detached retina. However, none of the eyes showed the foveal IS/OS line to be complete in the area overlying the focal serous retinal detachment.

Immediately after resolution of the retinal detachment, the IS/OS line was seen in many cases in the reattached retina; in $53(71.6 \%)$ of the 74 eyes, the IS/OS line was detected clearly beneath the fovea immediately after resolution of the retinal 
detachment. These eyes had relatively good VA at that time, with VA ranging from 0.7 to 1.5 (median, 1.2$)$. In the remaining 21 eyes $(28.4 \%)$, however, the IS/OS line could not be detected beneath the fovea shortly after resolution of the detachment. While VA of these eyes was variable at that time, and ranged from 0.1 to 1.5 (median,

5 0.9), mean VA in eyes without a foveal IS/OS line $(0.196 \pm 0.331$ in logMAR) was significantly poorer than that in eyes with a foveal IS/OS line $(-0.109 \pm 0.108$ in $\log$ MAR; $P<0.001, t$-test). Of these 21 eyes, 7 were in the acute phase, 6 were chronic, and 8 had a recurrence. The IS/OS line could be detected beneath the fovea more frequently in eyes with acute $\operatorname{CSC}\left(P=0.0129, X^{2}\right.$ test). of resolution of the serous retinal detachment (Table 1). The mean follow-up period after obtaining the reattachment was $11.6 \pm 11.7$ months. In four eyes, the IS/OS line was not detected beneath the fovea throughout the follow-up period. In these eyes, VA immediately after obtaining reattachment was poor, ranging from 0.1 to 0.9 (median. 0.225). These eyes did not show VA improvement during the follow-up $(P=$ 0.685 , paired $t$-test); median final VA of these four eyes was 0.15 (Figure 1).

During follow-up, however, nine eyes showed recovery of the foveal IS/OS line (Figures 2 and 3). The period until restoration of the foveal IS/OS line ranged from 2 to 16 months (median, 4 months). VA of these eyes immediately after resolution of 
the serous detachment was variable, ranging from 0.4 to 1.2 (median, 0.9). However, mean VA was significantly improved after restoration of the foveal IS/OS $(P=0.0027$, paired $t$-test). Mean final VA in these eyes was significantly better than that in eyes that showed the no recovery of the foveal IS/OS line $(P=0.0001, t$-test $)$.

$5 \quad$ In six of these nine eyes, although the foveal outer photoreceptor layer was indistinct immediately after resolution of the retinal detachment, VA at that time was good, ranging from 0.7 to 1.2 (median, 0.95) (Figure 2). However, three eyes showed a large defect of outer segments of foveal photoreceptors after resolution of the retinal detachment and poor VA, ranging from 0.4 to 0.5 (median, 0.4). During 10 follow-up, these three eyes showed restoration of the outer segments of foveal photoreceptors and achieved recovery of VA, which ranged from 0.8 to 1.5 (median, 1.0) (Figure 3). In two eyes, prognosis of the defect in the foveal IS/OS was not evaluated because of the recurrence of a serous detachment. 


\section{Discussion}

Increasingly, reports have shown the importance of the IS/OS line as a hallmark of integrity of the outer photoreceptor layer. ${ }^{6,9,11-17}$ OCT reflections from the IS/OS are

5 thought to arise from the abrupt change in the optical index of refraction at the boundary between the inner segments and the highly organized structure of the outer segments. $^{7}$ In eyes with active CSC, however, the IS/OS line could not be detected in the detached retina. ${ }^{6}$ Ko et al $^{18}$ suggest that the membranous stack of the photoreceptor segment is no longer perpendicular to the incoming OCT beam, and

10 the highly backreflecting signal at the IS/OS seems to be absent in detached retina. ${ }^{18}$ In eyes with active CSC, disappearance of the IS/OS line may not always indicate that the photoreceptor segments are substantially destroyed. Accordingly, even if an eye with active CSC does not show a distinct IS/OS line beneath the fovea, good VA is obtained often when the subretinal fluid is resorbed. fovea immediately after resolution of the serous detachment. In 4 of these 15 eyes, the IS/OS line was not detected beneath the fovea throughout the follow-up period, with concomitant poor vision. Our finding is supported by previous reports using Stratus OCT, in which integrity of the foveal photoreceptors after resolution of CSC is 
involved in final VA. ${ }^{3-5}$ More precisely, a recent study with spectral-domain OCT found that a large defect of the outer segments of the foveal photoreceptor layer was correlated with poor VA in eyes with resolved CSC. ${ }^{6}$ Photoreceptor cells are expected to be damaged when the retina is separated from the retinal pigment

5 epithelium and the choriocapillaris, their source of oxygen and nutrients. ${ }^{19,20}$ Recently, Matsumoto et $\mathrm{al}^{11}$ reported that the photoreceptor outer segments of the detached retina were elongated because of the lack of phagocytosis by the retinal pigment epithelium. In that report, prolonged detachment in CSC caused the decreased thickness of the outer nuclear layer due to apoptosis of the photoreceptor

10 cells. $^{11}$ Moreover, a steady decline in the thickness of the outer nuclear layer was shown in an animal model of experimental retinal detachment. ${ }^{21,22}$ During the active phase of CSC, irreversible damage in the foveal photoreceptor layer may well lead to the poor visual prognosis.

However, nine eyes in the study described herein showed recovery of the foveal

15 IS/OS line during follow-up. These eyes achieved a good final VA, even though VA was variable when the foveal IS/OS line was indistinct. Restoration of the outer foveal photoreceptor layer may be one possible explanation of this visual recovery. In six of these nine eyes, although the IS/OS line was indistinct immediately after resolution of the retinal detachment, all eyes showed relatively good VA at that time. 
In these eyes, function of the foveal photoreceptor cells may not be reduced markedly, even though the OCT beam did not cause a highly backscattering signal at the IS/OS junction because of some structural change in the photoreceptors. One possible explanation is that the membranous stack of photoreceptors shortly after

5 reattachment might be slightly irregular, and not perpendicular to the incoming OCT beam. Another possibility is that the photoreceptor layer was still swollen at that time. Previously, lida et $\mathrm{al}^{23}$ reported an increase in the thickness of retina that was detached due to active CSC. Recently, it has been reported that eyes in the acute phase of multiple evanescent white dot syndrome show an indistinct foveal IS/OS line

10 due to inflammatory swelling of the photoreceptors. ${ }^{24,25}$ In these eyes, however, the IS/OS line becomes obvious after resolution of the inflammation.

In the other three eyes, although VA was poor when the foveal IS/OS line was indistinct, they showed recovery of the IS/OS line and achieved a good VA during follow-up. In such eyes, while outer segments of the foveal photoreceptor layer 15 might be degenerated, the photoreceptor cells themselves may not be damaged lethally at that time, and may subsequently restore the outer segments. Using an animal model, it has been reported that the outer segments of the photoreceptor layer can show regrowth when the inner segments are not damaged, although it takes a very long time for them to regain their pre-detachment length. ${ }^{20}$ Recently, restoration 
of the IS/OS line has been described in various macular diseases. ${ }^{15,24,25}$ Spaide et al $^{15}$ reported that eyes with acute zonal occult outer retinopathy show disappearance of the IS/OS line, but that partial reconstruction of the IS/OS line is seen subsequently. Recently, Sano et $\mathrm{al}^{14}$ and Chang et $\mathrm{al}^{16}$ reported restoration of the outer

5 photoreceptor layer and visual recovery after closure of a macular hole. Ojima et al ${ }^{17}$ reported previously that eyes with resolved CSC often show a focal area with defects of the IS/OS with reduced retinal sensitivity, but that such functional changes might result ultimately in recovery.

In conclusion, in eyes with resolved CSC, it is generally thought that a large defect

10 of the outer segments of the foveal photoreceptors is correlated with poor visual outcome. $^{6}$ However, our finding suggest that when the IS/OS line is not detected beneath the fovea immediately after the resolution of active CSC, many affected eyes can be expected to show restoration of the IS/OS junction over time. Eyes with good VA at resolution of the CSC, even those with absence of the IS/OS line, probably have 15 well-preserved foveal photoreceptor cells and are most likely to show restoration. Eyes with resolved CSC that have poor VA, however, often show no restoration of the IS/OS line, and have a poor visual prognosis. Therefore, OCT findings immediately after resolution of active CSC may reveal only a step in reconstruction of the disorganized or damaged photoreceptor cells. 
Limitations of the current study are, of course, its retrospective nature, its small sample size, and the lack of follow-up in some patients. In addition, the examinations during the active phase of CSC were often performed with time-domain OCT, so we could not predict the final IS/OS condition or visual prognosis from the

5 CSC images obtained during the active phase of CSC. Furthermore, although the current study consisted of 74 eyes with CSC, only 21 eyes showed an indistinct IS/OS line beneath the fovea shortly after resolution of the serous retinal detachment. Further prospective study with larger sample size is necessary to elucidate more clearly the relationship between the condition of the foveal outer photoreceptor layer and visual function after resolution of active CSC. 


\section{References}

1. Spaide RF. Central serous chorioretinopathy. In: Holz FG, Spaide RF ,editors.

Medical Retina. Berlin, Heidelberg: Springer; 2004. p. 77-93.

5 2. Cardillo Piccolino F, Eandi CM, Ventre L, et al. Photodynamic therapy for chronic central serous chorioretinopathy. Retina 2003;23:752-763.

3. Eandi CM, Chung JE, Cardillo-Piccolino F, Spaide RF. Optical coherence tomography in unilateral resolved central serous chorioretinopathy. Retina $2005 ; 25: 417-421$.

4. Piccolino FC, de la Longrais RR, Ravera G, et al. The foveal photoreceptor layer and visual acuity loss in central serous chorioretinopathy. Am J Ophthalmol 2005;139:87-99.

5. Wang MS, Sander B, Larsen M. Retinal atrophy in idiopathic central serous chorioretinopathy. Am J Ophthalmol 2002;133:787-793. photoreceptor layer in central serous chorioretinopathy using high-speed optical coherence tomography. Ophthalmology 2007;114:2197-2207.

7. Drexler W, Sattmann H, Hermann B, et al. Enhanced visualization of macular pathology with the use of ultrahigh-resolution optical coherence tomography. 
Arch Ophthalmol 2003;121:695-706.

8. Ko TH, Fujimoto JG, Schuman JS, et al. Comparison of ultrahigh- and standard-resolution optical coherence tomography for imaging macular pathology. Ophthalmology 2005;112:1922-1935.

5 9. Hangai M, Ojima Y, Gotoh N, et al. Three-dimensional imaging of macular holes with high-speed optical coherence tomography. Ophthalmology 2007;114:763-773.

10. Maruko I, lida T, Sekiryu T, Saito M. Morphologic changes in the outer layer of the detached retina in rhegmatogenous retinal detachment and central serous chorioretinopathy. Am J Ophthalmol 2009;147:489-494.

11. Matsumoto H, Kishi S, Otani T, Sato T. Elongation of photoreceptor outer segment in central serous chorioretinopathy. Am J Ophthalmol 2008;145:162-168.

12. Fujimoto $\mathrm{H}$, Gomi F, Wakabayashi T, et al. Morphologic changes in acute central serous chorioretinopathy evaluated by Fourier-domain optical coherence tomography. Ophthalmology 2008;115:1494-1500.

13. Costa RA, Calucci D, Skaf M, et al. Optical coherence tomography 3: Automatic delineation of the outer neural retinal boundary and its influence on retinal thickness measurements. Invest Ophthalmol Vis Sci 2004;45:2399-2406. 
14. Sano M, Shimoda $Y$, Hashimoto H, Kishi S. Restored photoreceptor outer segment and visual recovery after macular hole closure. Am J Ophthalmol 2009; 147:313-318.

15. Spaide RF, Koizumi H, Freund KB. Photoreceptor outer segment abnormalities as a cause of blind spot enlargement in acute zonal occult outer retinopathy-complex diseases. Am J Ophthalmol 2008;146:111-120.

16. Chang LK, Koizumi H, Spaide RF. Disruption of the photoreceptor inner segment-outer segment junction in eyes with macular holes. Retina 2008;28:969-975.

17. Ojima $Y$, Tsujikawa $A$, Hangai $M$, et al. Retinal sensitivity measured with the Micro Perimeter 1 after resolution of central serous chorioretinopathy. Am J Ophthalmol 2008;146:77-84.

18. Ko TH, Fujimoto JG, Duker JS, et al. Comparison of ultrahigh- and standard-resolution optical coherence tomography for imaging macular hole pathology and repair. Ophthalmology 2004;111:2033-2043.

19. Arroyo JG, Yang L, Bula D, Chen DF. Photoreceptor apoptosis in human retinal detachment. Am J Ophthalmol 2005;139:605-610.

20. Lewis GP, Charteris DG, Sethi CS, Fisher SK. Animal models of retinal detachment and reattachment: identifying cellular events that may affect visual 
recovery. Eye 2002;16:375-387.

21. Erickson PA, Fisher SK, Anderson DH, et al. Retinal detachment in the cat: the outer nuclear and outer plexiform layers. Invest Ophthalmol Vis Sci 1983;24:927-942.

5 22. Wilson DJ, Green WR. Histopathologic study of the effect of retinal detachment surgery on 49 eyes obtained post mortem. Am J Ophthalmol 1987;103:167-179.

23. lida T, Hagimura N, Sato T, Kishi S. Evaluation of central serous chorioretinopathy with optical coherence tomography. Am J Ophthalmol 2000;129:16-20.

24. Kanis MJ, van Norren D. Integrity of foveal cones in multiple evanescent white dot syndrome assessed with OCT and foveal reflection analyser. $\mathrm{Br} \mathrm{J}$ Ophthalmol 2006;90:795-796.

25. Sikorski BL, Wojtkowski M, Kaluzny JJ, et al. Correlation of spectral optical coherence tomography with fluorescein and indocyanine green angiography in multiple evanescent white dot syndrome. Br J Ophthalmol 2008;92:1552-1557. 


\section{Figure legends}

Figure 1. Visual acuity (VA) in eyes with a large defect of the junctions between inner and outer segments of the photoreceptors (IS/OS) beneath the fovea

5 immediately after resolution of central serous chorioretinopathy. Blue diamonds, eyes that showed recovery of IS/OS beneath the fovea during the follow-up; red diamonds, eyes that showed no recovery of IS/OS beneath the fovea throughout follow-up; yellow diamonds, eyes that showed a recurrence of central serous chorioretinopathy after initial resolution.

Figure 2 A-F. Indistinct line of junctions between inner and outer segments of the photoreceptors (IS/OS) beneath the fovea immediately after resolution of central serous chorioretinopathy (CSC) with good visual acuity. A 56-years-old man had a one-month history of visual disturbance in the right eye due to active CSC. A Fundus

15 photograph at the initial visit shows focal serous retinal detachment involving the fovea. Visual acuity of the right eye was 1.2 on a Landolt chart. B Fluorescein angiography shows pinpoint leakage. C Indocyanine green angiography also shows a hyperfluorescent spot. D Horizontal sectional image obtained with optical coherence tomography (OCT) at the initial visit shows a focal serous retinal 
detachment. E One month after the initial visit, serous retinal detachment has resolved completely with no treatment. A horizontal image obtained with OCT shows a large defect of the IS/OS beneath the fovea. However, visual acuity of the right eye was 1.0. F Three months after resolution of the retinal detachment, OCT confirms

5 complete recovery of the IS/OS beneath the fovea. Visual acuity of the right eye was 1.5.

Figure 3A-F. Indistinct line of junctions between inner and outer segments of the photoreceptors (IS/OS) beneath the fovea immediately after resolution of central serous chorioretinopathy (CSC) with poor visual acuity. A 51-years-old man had a three-month history of central scotoma in the left eye due to active CSC. A Fundus photograph at the initial visit shows focal serous retinal detachment involving the fovea. Visual acuity of the left eye was 1.2 on a Landolt chart. B Fluorescein angiography shows pinpoint leakage. C Indocyanine green angiography shows an

15 area of blockade of the fluorescein due to the presence of subretinal fluid. D A horizontal sectional image obtained with optical coherence tomography (OCT) at the initial visit shows a focal serous retinal detachment. E Six months after the initial visit, serous retinal detachment has resolved completely, without any treatment. However, visual acuity of the left eye had decreased to 0.5 , and a horizontal image obtained with 
OCT now shows a large defect of the junctions between inner and outer segments of

the photoreceptors (IS/OS) beneath the fovea. F Three months after resolution of

the retinal detachment, OCT confirms complete recovery of the IS/OS junctions

beneath the fovea; visual acuity of the left eye had improved to 1.5 . 
Table 1. Eyes That Did Not Show IS/OS Line Beneath the Fovea Immediately After Resolution of a Serous Retinal Detachment Associated With Central Serous Chorioretinopathy

\begin{tabular}{|c|c|c|c|c|c|c|c|c|c|c|c|c|c|}
\hline \multirow[b]{2}{*}{ Patient } & \multirow[b]{2}{*}{$\begin{array}{c}\text { Age/gende } \\
r\end{array}$} & \multirow[b]{2}{*}{ Phase } & \multirow[b]{2}{*}{$\begin{array}{c}\text { Duration of } \\
\text { Symptoms } \\
\text { (Mo.) }\end{array}$} & \multicolumn{2}{|c|}{ At Initial Visit } & \multirow[b]{2}{*}{ Treatment } & \multirow{2}{*}{$\begin{array}{c}\text { Duration } \\
\text { of Retinal } \\
\text { Detach- } \\
\text { ment } \\
\text { (Mo.) }\end{array}$} & \multicolumn{2}{|c|}{$\begin{array}{l}\text { At the Resolution of } \\
\text { Retinal Detachment }\end{array}$} & \multirow[b]{2}{*}{$\begin{array}{l}\text { Restoration } \\
\text { of IS/OS }\end{array}$} & \multirow{2}{*}{$\begin{array}{l}\text { Period Till } \\
\text { Restoration } \\
\text { of IS/OS } \\
\text { (Mo.) }\end{array}$} & \multicolumn{2}{|c|}{ At Final Visit } \\
\hline & & & & $\begin{array}{l}\text { Visual } \\
\text { acuity }\end{array}$ & $\begin{array}{c}\text { Foveal } \\
\text { thickness } \\
(\mu \mathrm{m})\end{array}$ & & & $\begin{array}{l}\text { Visual } \\
\text { acuity }\end{array}$ & $\begin{array}{c}\text { Foveal } \\
\text { thickness } \\
(\mu \mathrm{m})\end{array}$ & & & $\begin{array}{l}\text { Visual } \\
\text { acuity }\end{array}$ & $\begin{array}{c}\text { Foveal } \\
\text { thickness } \\
(\mu \mathrm{m})\end{array}$ \\
\hline 1 & $42 / \mathrm{M}$ & Chronic & 15 & 0.2 & 232 & - & 12 & 0.15 & 149 & No & - & 0.15 & 151 \\
\hline 2 & $65 / M$ & Chronic & 7 & 0.7 & - & PC, PDT & 44 & 0.3 & 152 & No & - & 0.15 & 204 \\
\hline 3 & $57 / M$ & Chronic & 36 & 0.06 & 303 & - & 2 & 0.1 & 192 & No & - & 0.15 & 189 \\
\hline 4 & $66 / M$ & Chronic & 7 & 0.6 & 321 & - & 1.5 & 0.9 & 149 & No & - & 0.8 & 158 \\
\hline 5 & $65 / M$ & Recurrence & 48 & 0.3 & 232 & PC, PDT & 2 & 0.4 & 141 & Yes & 10 & 1.0 & 175 \\
\hline 6 & $38 / M$ & Recurrence & 1 & 0.8 & 231 & - & 2 & 0.4 & 121 & Yes & 4 & 0.8 & 161 \\
\hline 7 & $51 / M$ & Acute & 3 & 1.2 & 421 & - & 6 & 0.5 & 215 & Yes & 3 & 1.5 & 189 \\
\hline 8 & $49 / M$ & Recurrence & 12 & 0.8 & 269 & PC & 18 & 0.7 & 182 & Yes & 2 & 1.2 & 211 \\
\hline 9 & $55 / M$ & Recurrence & 60 & 0.3 & 357 & PC, PDT & 1.5 & 0.9 & 179 & Yes & 16 & 1.2 & 260 \\
\hline 10 & $60 / M$ & Chronic & 36 & 0.7 & 349 & - & 3 & 0.9 & 173 & Yes & 3 & 1.0 & 161 \\
\hline 11 & $45 / M$ & Recurrence & 3 & 0.9 & 535 & - & 2.5 & 1.0 & 182 & Yes & 6 & 1.2 & 198 \\
\hline 12 & $43 / F$ & Recurrence & 0.3 & 1.2 & 536 & - & 4 & 1.0 & 210 & Yes & 12 & 1.5 & 134 \\
\hline 13 & $56 / M$ & Recurrence & 1 & 1.2 & 464 & - & 1 & 1.2 & 143 & Yes & 3 & 1.5 & 198 \\
\hline 14 & $70 / F$ & Recurrence & 0.1 & 0.6 & 588 & - & 1.5 & 0.9 & 199 & Recurrence & - & 0.9 & 248 \\
\hline 15 & $52 / M$ & Acute & 4 & 0.6 & 206 & - & 4 & 1.2 & 221 & Recurrence & - & 1.2 & 277 \\
\hline
\end{tabular}

IS/OS, junctions between inner and outer segments of the photoreceptors; M, male; F, female; Mo, months; PC, photocoagulation; PDT, photodynamic therapy. 
$\mathbf{F}$ 京都大学

КYOTO UNIVERSITY)Wnload high resolution image

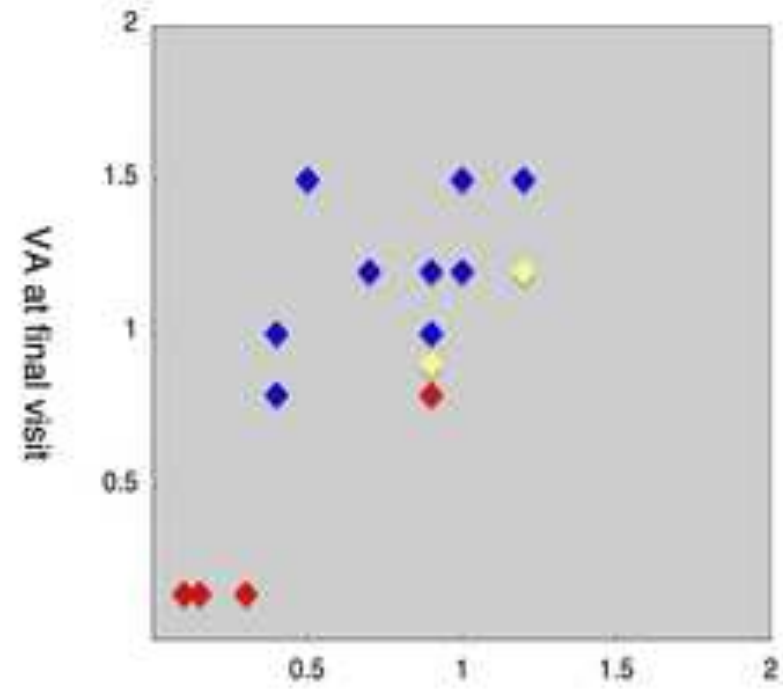

VA immediately after resolution of CSC 
F: 京都大学

C KYTO UNIVERSITY)Wnload high resolution image

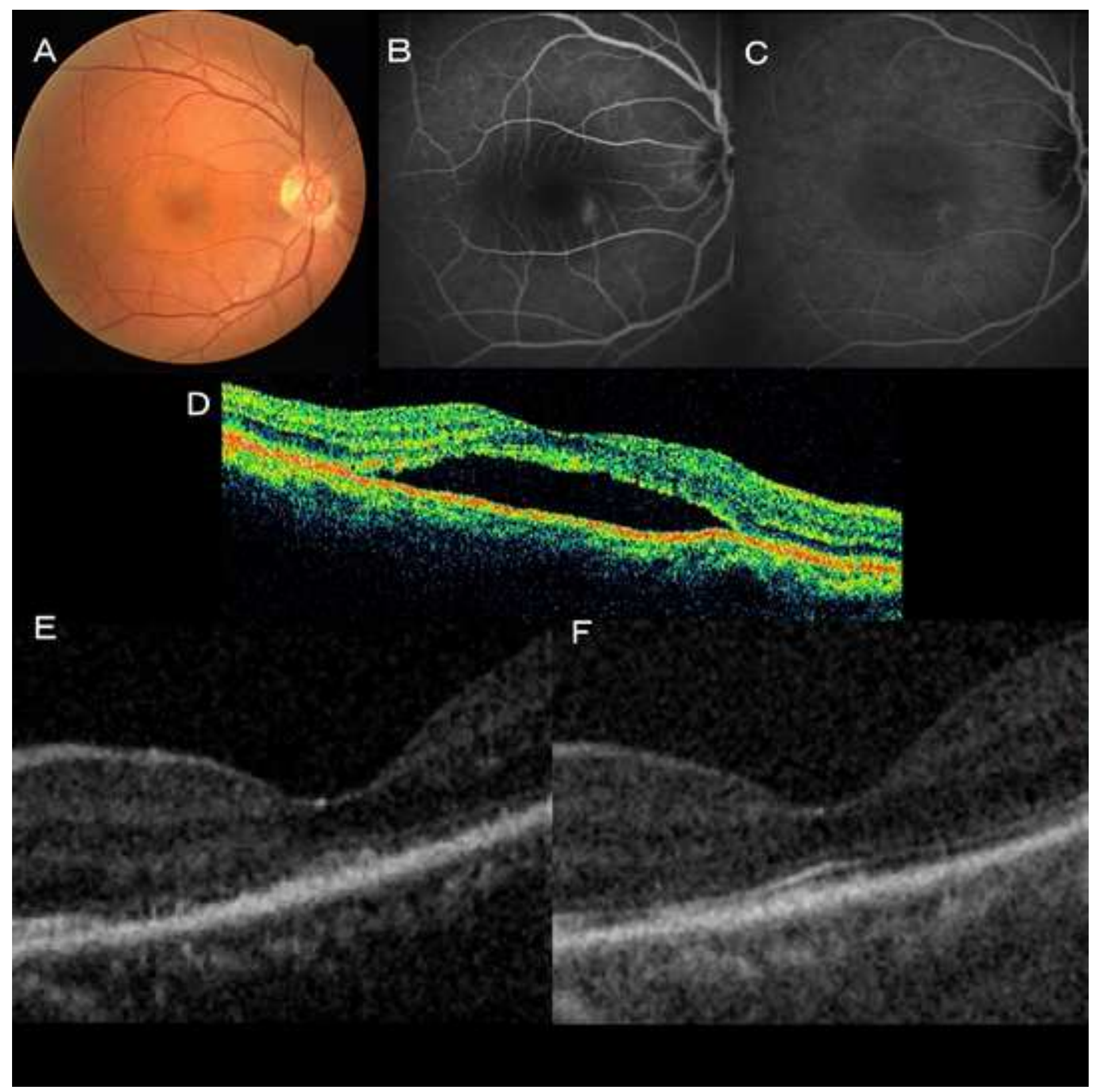

D 5.

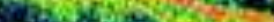

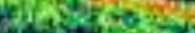

G

$E$ seritents

B
A Self-archived copy in
Kyoto University Research Information Repository (1) 
F

A Self-archived copy in

( күото uNIVERSITY)Wnload high resolution image

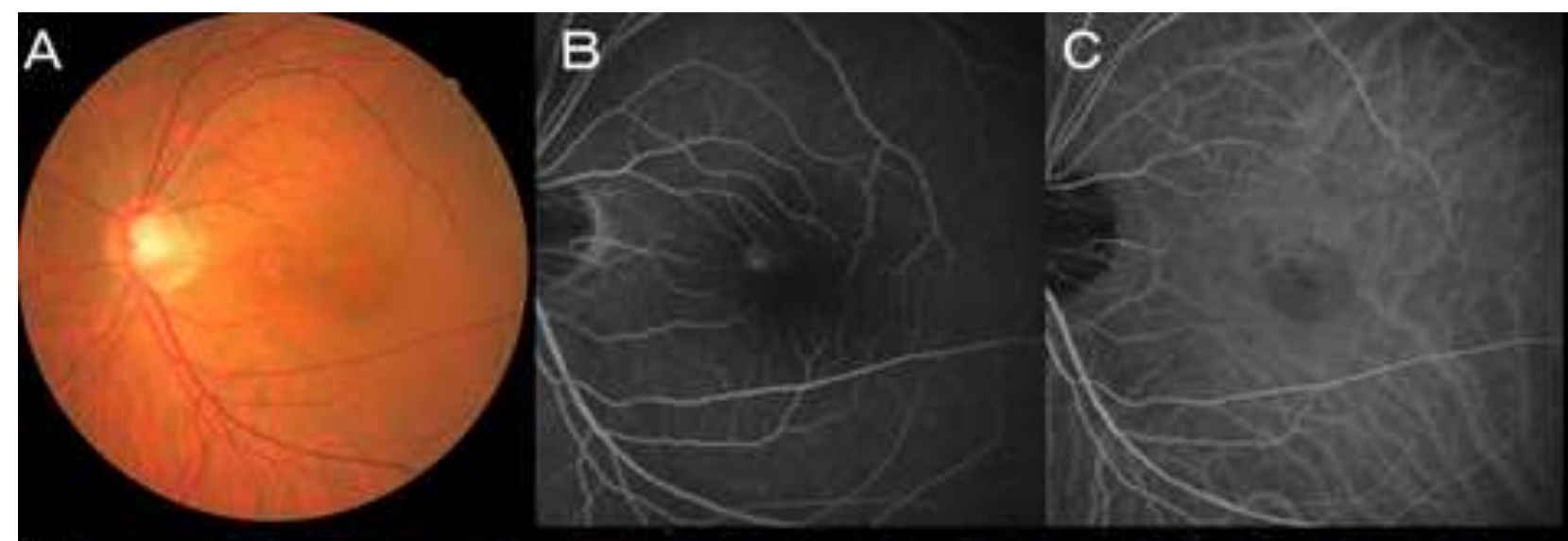

D

E

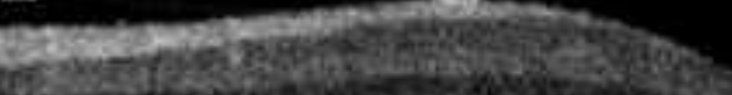

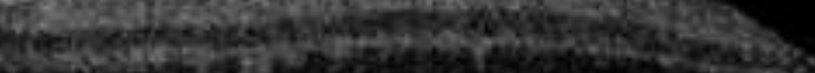

4.

F

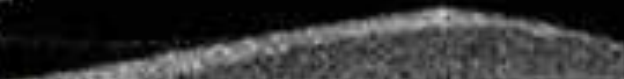

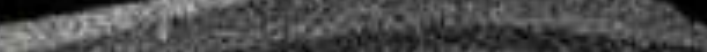

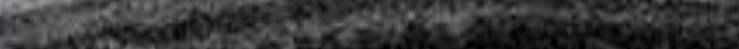

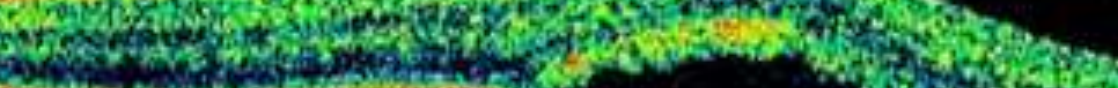

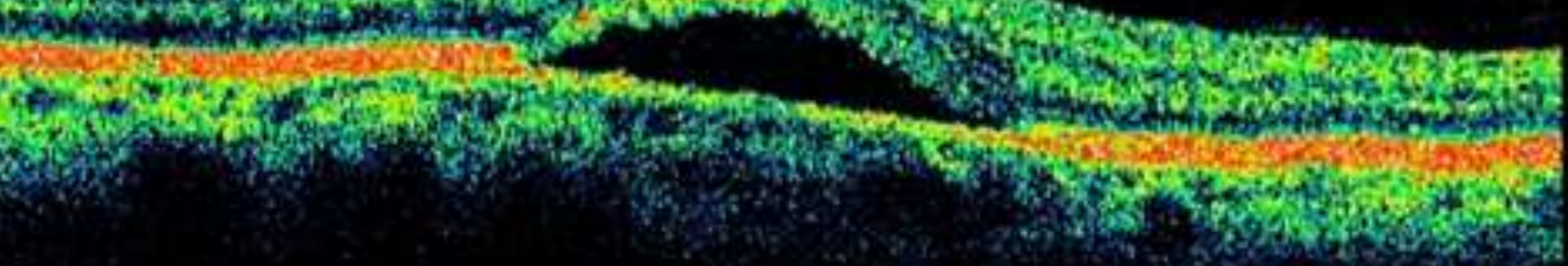

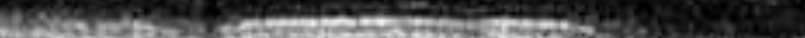

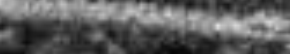

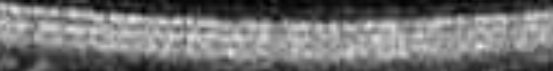

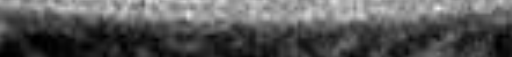

Wh

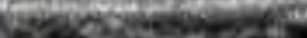

